\title{
Obesity in Kenya: A Synthesis of the Literature
}

\author{
Maraga Edith* \\ Department of Community Dentistry and Periodontology, School of Dentistry, Kenya
}

*Corresponding author: Maraga Edith, Assistant Lecturer, Department of Community Dentistry and Periodontology, School of

Dentistry, Eldoret, Kenya

\begin{abstract}
ARTICLE INFO
Received: 幽 September 30, 2019

Published: 幽 October 15, 2019

Citation: Maraga Edith. Obesity in Kenya: A Synthesis of the Literature. Biomed J Sci \& Tech Res 22(1)-2019. BJSTR. MS.ID.003680.
\end{abstract}

Abbreviations: BMI: Body Mass Index; WHO: World Health Organisation; NCDs: Non- Communicable Diseases

\section{ABSTRACT}

Background: Obesity has become a worrying trend in Kenya as well as other parts of the world. The environment, culture and poor healthcare systems have contributed to this rise.

Methods: a desktop technique was used to ascertain the articles on obesity in Kenya by use of PubMed and hand-searching.

Results: Obesity was reported to be highest amongst urban dwellers with no gender preference.

Conclusion: measures to curb and reduce obesity have been suggested to take place not only at the individual level, but also at the societal level.

Keywords: Obesity; Kenya

\section{Background}

The prevalence of obesity in Kenya has risen in the past decade and this is causing a worrying situation. Various factors have been suggested to cause this rise, namely; the environment, culture and poor health care system. This review aims at describing the factors that cause the increase in the prevalence of obesity, analyses the public health responses and provides recommendations. Several chronic diseases are associated with overweight and obesity, namely diabetes, cardiovascular diseases and cancer. In the past, obesity was only a problem in high income countries but is now dramatically on the rise in low-and middle- income counties, especially in urban settings [1]. Worldwide obesity has more than doubled since 1980. In 2008, 1.5 billion adults, 20 years and older were overweight. Of these over 200 million men and nearly 300 million women were obese. $65 \%$ of the world's population live in countries where overweight and obesity kills more people than underweight. Nearly 43 million children under the age of five were overweight in 2010. Obesity is preventable [1]. Overweight and obesity are the fifth leading risk for global deaths. At least 2.8 million adults die each year as a result of being overweight or obese. In addition, $44 \%$ of the diabetes burden, $23 \%$ of the ischaemic heart disease burden and between $7 \%$ and $41 \%$ of certain cancer burdens are attributable to overweight and obesity [1].

\section{Methods}

A desktop technique was used to ascertain the articles on obesity in Kenya by use of PubMed and hand-searching. A critical analysis was done to synthesis the articles obtained.

\section{Results}

\section{Health Determinants}

The causes of death from obesity today are different from those of the past. But many low- and middle-income countries are facing a "double burden" of disease, as they continue to deal with the problems of infectious disease and under-nutrition, they are experiencing a rapid upsurge in non-communicable disease risk factors such as obesity and overweight, particularly in urban settings [1]. No one factor causes obesity. There are several factors that lead to the condition including biological, psychological, behavioural, social and environment determinants. To illustrate the issues in Kenya some of the elements of the Evans and Stoddart model will be used as a reference point for discussion. This model [2] argues that genetic endowment, social and physical environment directly interact with the individual, causing responses that may cause disease (obesity), and affect well-being or health and function. This 
model being the most recent and complex explains the complexity of health and that the individual also has power to influence their environment.

\section{Environment}

People migrate from rural areas to settle in the urban setting to seek employment and possibly better health care. For the situation in Kenya, the employment opportunities afforded in the urban setting, create an increase of income, leading to an improvement in well-being which results in the individual response which may be positive (health and function) or negative (disease being obesity). However, due to the demands of urban living that leads to a sedentary lifestyle, the amount of exercise reduces, as stated by Onywera [3]. This is regardless of the level of education, socio-economic status or behaviour, resulting in obesity. Obesity appeared to be prevalent in women in the high-income group (7278 kJ) in urban areas (7049 $\mathrm{kJ}$ ) with $34.5 \%$ total fat intake than in rural areas $29.7 \%$ of energy.

\section{Obesogenic Environment}

One cause of obesity and overweight is an energy imbalance between calories consumed and calories expended. Globally, there has been: an increased intake of energy-dense foods that are high in fat, salt and sugars but low in vitamins, minerals and other micronutrients [1]; a decrease in physical activity due to the increasingly sedentary nature of many forms of work, changing modes of transportation, and increasing urbanisation. Changes in dietary and physical activity patterns are often the result of environmental and societal changes associated with development and lack of supportive policies in sectors such as health, agriculture, transport, urban planning, environment, food processing, distribution, marketing and education [1]. The cheap, highly processed foods and fatty diets in Kenya have been attributed to the marked "nutritional transition" [2] which occurred over the past 400 years since the colonial times [2]. This moved the whole population from the traditional and indigenous foods that are associated with health benefits [2]

\section{Culture}

In Kenya, and Africa at large, some socio-cultural beliefs view obesity and overweight as an admired trait. It is seen as a sign of prestige and wealth [3] There is a lack of strong and outward looking social networks which have been shown to be protective of health [2]

\section{Health Care}

Depending on whether the health care system is well structured, it is then involved in improving health and leading to either prosperity or an individual response to health and function. Hsia et al [4] in a study to access to health care in Sub-Saharan Africa recognised the critical "infrastructure gaps" that obstruct the health systems. Okeyo et al [5] speculated that overweight and obesity, with their related disease conditions will constitute a major threat to the economically productive adults causing a huge health-care burden soon in developing countries.

\section{Discussion}

Public health responses to this issue should focus on interventions that include changes to the major determinants of obesity. Adopted by the World Health Assembly in 2004, the WHO Global Strategy on Diet, Physical Activity and Health describes the actions needed to support healthy diets and regular physical activity. The Strategy calls upon all stakeholders to act at global, regional and local levels to improve diets and physical activity patterns at the population level [1]. WHO developed the 2008-2013 Action plan for the global strategy for the prevention and control of non-communicable diseases (NCDs) to help the millions who are already affected cope with these lifelong illnesses and prevent secondary complications? This action plan aimed to build on, the WHO Framework Convention on Tobacco Control and the WHO Global Strategy on Diet, Physical Activity and Health.

The action plan provides a roadmap to establish and strengthen initiatives for the surveillance, prevention and management of NCDs. Overweight and obesity, as well as their related non-communicable diseases, are largely preventable. Supportive environments and communities are fundamental in shaping people's choices, making the healthier choice of foods and regular physical activity the easiest choice, and therefore preventing obesity.

At the individual level, people can:

a) Limit energy intake from total fats;

b) Increase consumption of fruit and vegetables, as well as legumes, whole grains and nuts;

c) Limit the intake of sugars;

d) Engage in regular physical activity;

e) Achieve energy balance and a healthy weight.

Individual responsibility can only have its full effect where people have access to a healthy lifestyle. Therefore, at the societal level it is important to:

a) Support individuals in following the recommendations above, through sustained political commitment and the collaboration of many public and private stakeholders;

b) Make regular physical activity and healthier dietary patterns affordable and easily accessible to all - especially the poorest individuals.

c) Might also include the provision of school meals and health education of not only the school children but the community at large.

The food industry can play a significant role in promoting healthy diets by: 
a) Reducing the fat, sugar and salt content of processed foods;

b) Ensuring that healthy and nutritious choices are available and affordable to all consumers;

c) Practicing responsible marketing;

d) Ensuring the availability of healthy food choices and supporting regular physical activity practice in the workplace.

Initiatives to improve the situation of obesity should therefore be at the individual, family, community, population and societal levels. There appeared to be no published literature on the initiatives to improve the obesity epidemic in Kenya and Africa at large.

\section{Recommendations}

1. Interdisciplinary perspective at various societal levels, [6-9] involving, research, education, direct management in primary health care is required to improve the obesity situation in Kenya. This could be achieved by involving the incorporation of food and nutrition studies into different areas such as the primary school curriculum, which might affect a shift of behaviours, attitudes, culture, values and beliefs to benefit the community at large $[9,10]$.

2. Incentives for healthy eating habits [8] should be developed to nationally encourage consumption of traditional foods and the consumption of high fat modern food in moderation.

3. The promotion of strategies to increase the level [10-12] of physical activity in the urban population both at local and national levels.

4. Improved surveillance and monitoring [8-10] of the population's health and well-being is essential. This may be achieved by the Kenyan government improving the infrastructure in the country.

ISSN: 2574-1241

DOI: 10.26717/BJSTR.2019.22.003680

Maraga Edith. Biomed J Sci \& Tech Res

(C) (9) This work is licensed under Creative (c) ${ }_{\mathrm{BY}}$ Commons Attribution 4.0 License

Submission Link: https://biomedres.us/submit-manuscript.php

\section{Conclusion}

There is an increase in obesity especially in the urban settings in Kenya. This has been attributed to the rural to urban migration due to better employment opportunities and improved health care. However, due to the sedentary lifestyles, decreased amount of exercise and consumption of high-calorie dense foods, obesity develops.

\section{References}

1. (2011) WHO.

2. Evans RG, Stoddart GL (1994) Producing health, consuming health care In: Evans RG, Barer ML, Marmor TR (Eds.), Why are some people healthy and others are not? The determinants of health of populations. Berlin, Walter de Gruyter, New York, USA, pp. 27-64.

3. Onywera VO (2010) Childhood obesity and physical inactivity threat in Africa: strategies for a healthy future. Glob Health Promot 17(2): 45-46.

4. Hsia RY, Mbembati NA, Macfarlane S, Kruk ME (2011) Access to emergency and surgical care in sub-Saharan Africa: the infrastructure gap. Health Policy Plan 27(3): 234-244.

5. Okeyo OD, Ayado OL, Mbagaya GM (2009) Physical activity and dietary fat as determinants of body mass index in a cross-sectional corelational design. East Afr J Public Health 6(1): 32-36.

6. Hilbert A, Ried J, Schneider D, Juttner C, Sosna M, et al. (2007) Primary prevention of adult obesity an interdisciplinary analysis Herz 32(7): 542-552.

7. Verduin P, Agarwal S, Waltman S (2005) Solutions to obesity: perspectives from the food industry. Am J Clin Nutr 82 (1 Suppl): 259S-261S.

8. Sargeant LA, Wilks RJ, Forrester TE (2001) Chronic diseases-facing a public health challenge. West Indian Med J 50(4): 27-31.

9. Mela DJ (2001) Determinants of food choice relationships with obesity and weight control. Obes Res 9(4): 249S-255S.

10. Szponar L, Ciok J, Dolna A, Oltarzewski M (2007) Policy options for responding to the growing challenge from obesity (PorGrow) in Poland. Obes Rev 8(2): 91-98.

11. Marr L (2004) Soft drinks, childhood overweight, and the role of nutrition educators: let's base our solutions on reality and sound science. J Nutr Educ Behav 36 (5): 258-265.

12. Floriani V, Kennedy C (2007) Promotion of physical activity in primary care for obesity treatment/prevention in children. Curr Opin Pediatr 19(1): 99-103.

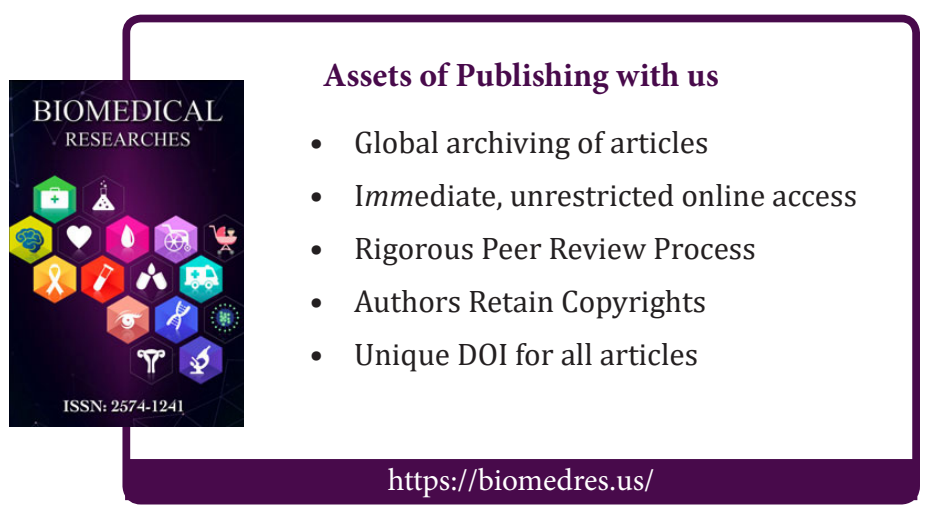

